\title{
Hepatitis C Virus Clearance by Direct-acting Antiviral Results in Rapid Resolution of Hepatocytic Injury as Indicated by Both Alanine Aminotransferase and Aspartate Aminotransferase Normalization
}

\author{
Tung Huynh, Johnathan Zhang and Ke-Qin Hu* \\ Division of Gastroenterology and Hepatology, School of Medicine, University of California, Orange, CA, USA
}

\begin{abstract}
Background and Aims: Hepatitis C virus (HCV) infection results in hepatocytic injury with elevation of both alanine aminotransferase (ALT) and aspartate aminotransferase (AST). It remains to be determined if direct-acting antiviral treatment can terminate hepatocytic injury following virologic response. To this end, we evaluated the pattern and predicting factors of ALT and AST normalization during and after direct-acting antiviral treatment with sustained virologic response at 12 weeks (SVR12). Methods: Single-center retrospective study on $115 \mathrm{HCV}$-infected patients who achieved SVR12 was performed. Results: At treatment week 2, 100\% and $45.9 \%$ showed decline in HCV RNA to $<700 \mathrm{IU} / \mathrm{mL}$ and undetectable levels, respectively, and this was associated with $85.5 \%, 83.9 \%$ and $77.4 \%$ ALT normalization, AST normalization and ALT and AST normalization. At end of treatment, $85.6 \%$ of patients with baseline elevation of both ALT and AST had normalization of both ALT and AST. At posttreatment weeks 12 and $24,90.8 \%$ and $94.8 \%$ had normalization of both ALT and AST. HCV clearance also resulted in further decline of both ALT and AST in those with baseline <40 IU. Univariate analysis showed baseline Child-Pugh score of $<6$, model for end-stage liver disease score of $<10$, HCV genotype 1 , and HCV RNA of $<500 \mathrm{IU} / \mathrm{mL}$ at treatment week 2 were associated with sustained normalization of both ALT and AST at posttreatment week 12 . On multivariate analysis, baseline model for end-stage liver disease score of $<10$ was significantly associated with normalization of both ALT and AST at posttreatment week 12, independent of baseline Child-Pugh score $<6$, HCV genotype 1 , and HCV RNA of $<500 \mathrm{IU} / \mathrm{mL}$ at treatment week 2. Conclusions: During direct-acting antiviral therapy, $85.5 \%$ and $83.9 \%$ had normalization of both ALT and AST as early as in week 2 , providing
\end{abstract}

Keywords: Chronic hepatitis C; Direct acting antiviral; ALT and AST normalization; Hepatocytic injury.

Abbreviations: AFP, alpha-fetoprotein; AIH, autoimmune hepatitis; ALT, alanine aminotransferase; AST, aspartate aminotransferase; $\mathrm{CHC}$, chronic hepatitis C; C-P, Child-Pugh; DAA, direct-acting antiviral; EOF, end of follow-up; EOT, end of treatment; GT, genotype; HCV, hepatitis C virus; MELD, model for end-stage liver disease; NASH, nonalcoholic steatohepatitis; PEG-IFN, pegylated-interferon; RBV ribavirin; SVR12, sustained virologic response at 12 weeks; ULN, upper limit of normal.

Received: 26 February 2018; Revised: 1 July 2018; Accepted: 23 August 2018 *Correspondence to: Ke-Qin Hu, Division of Gastroenterology and Hepatology, School of Medicine, University of California, 101 The City Drive, Building 56, Ste. 237, Orange, CA 92868, USA. Tel: +1-714-456-6926, Fax: +1-714-456-3283, E-mail:kqhu@uci.edu biochemical evidence of hepatocytic injury resolution. Sustained normalization of both ALT and AST was seen in $90.8 \%$ at posttreatment weeks 12 , and was independently associated with baseline model for end-stage liver disease score of $<10$.

Citation of this article: Huynh T, Zhang J, Hu KQ. Hepatitis $\mathrm{C}$ virus clearance by direct-acting antiviral results in rapid resolution of hepatocytic injury as indicated by both alanine aminotransferase and aspartate aminotransferase normalization. J Clin Transl Hepatol 2018;6(3):258-263. doi: 10.14218/ JCTH.2018.00014.

\section{Introduction}

Hepatitis C virus (HCV) infection affects approximately 115 million people worldwide and 3-5 million people in the USA. ${ }^{1,2}$ In most cases, HCV infection becomes chronic, which can result in chronic hepatitis $\mathrm{C}(\mathrm{CHC})$, cirrhosis and development of hepatocellular carcinoma. ${ }^{3}$ Interferon plus ribavirin (RBV) was once the mainstay regimen for HCV treatment, but was not effective and was associated with profound side effects. Since the first generation of directacting antiviral agents (DAAs)-boceprevir and telaprevirwere launched in 2011, significant advances have been made. The result has been clinical applications of more effective, safer, and newer generation of DAAs, starting in 2013, which revolutionized HCV treatment with superior sustained virologic response (SVR) rates, shorter treatment duration, and minimal side effects. ${ }^{4}$

To date, the USA Food and Drug Administration has approved the following DAA therapies: sofosbuvir (Sovaldi), simeprevir (Olysio), ledipasvir-sofosbuvir (Harvoni), ombitasvir-paritaprevir/ritonavir-dasabuvir (Viekira pak, Viekira XR), daclatasvir (Daklinza), ombitasvir-paritaprevir/ ritonavir (2D regimen) (Technivie), elbasvir-grazoprevir (Zepatier), sofosbuvir-velpatasvir (Epclusa), sofosbuvirvelpatasvir-voxilaprevir (Vosevi), and glecaprevir-pibrentasvir (Mavyret). In fact, these DAA regimens have become the standard of care in the USA, and many other countries. Consequently, HCV infection has become a curable disease, defined as SVR at 12 weeks (SVR12), i.e. the absence of detectable HCV RNA at 12 weeks after treatment completion. Studies have confirmed that SVR results in significantly improved long-term outcomes, including reduced morbidity and mortality in these patients. ${ }^{5,6}$ 
Both alanine aminotransferase (ALT) and aspartate aminotransferase (AST) have been biochemical surrogates for liver injury, including HCV-mediated hepatocytic injury. During chronic HCV infection, ALT and AST are frequently elevated. Although HCV RNA monitoring during HCV treatment has been a standard practice, ALT and AST monitoring is also important to assess improvement of liver injury, drug side effects or interactions, and treatment response. However, changes in early treatment and full dynamic patterns of ALT and AST during and post DAAs treatment-have not been well studied. Furthermore, patients with HCV infection often have concomitant nonalcoholic fatty liver disease or other nonviral liver disease, potentially confounding the liver enzyme elevation. It remains to be determined whether these coexisting liver diseases or other factors could impact sustained biochemical normalization in patients who achieve SVR following HCV DAA therapy.

The present study assessed the dynamics of AST and ALT in patients who received DAA treatment with resulting SVR12 and evaluated factors that predict sustained biochemical response or normalization of both AST and ALT in these patients.

\section{Methods}

\section{Patient enrollment}

This was a single-center retrospective study. Institutional Review Board approval was obtained, and informed consent was waived. Inclusion criteria included patients with $\mathrm{HCV}$, treated with DAAs with or without RBV, and having a minimum of 12 weeks posttreatment follow-up with confirmed SVR12. Exclusion criteria included patients with incomplete or missing lab data during treatment, completion of DAAs treatment but lack of SVR12 data, and completion of HCV treatment but relapse after DAA treatment. Patients with chronic HCV infection who received DAA treatment from $4 / 2014$ to $10 / 2016$ at the University of California Irvine Medical Center were assessed and enrolled if they met the above inclusion criteria.

A total of 149 charts were reviewed, leading to 28 patients being excluded - 19 due to incomplete or missing data during or after completed DAA treatment, and 9 patients due to completion of DAA treatment but no SVR12 data. There were 121 patients who completed the DAA treatment and had 12 week post treatment follow-up data. Six of those patients relapsed after completed treatment and were excluded. Consequently, 115 patients who achieved SVR12 were included in the analysis.

\section{Data collection}

Baseline data collection included age, sex, ethnicity, liver disease stage, comorbidities, presence of steatosis on imaging or biopsy, presence of nonalcoholic steatohepatitis (NASH) by biopsy, diagnosis of autoimmune hepatitis (AIH), diagnosis of cirrhosis, presence of decompensation, Child-Pugh (C-P) score, model for end-stage liver disease (MELD) score, baseline weight, body mass index, history of prior treatment experience, and previous treatment regimen if experienced. Baseline and follow-up lab data included HCV genotype (GT), HCV RNA PCR findings, international normalized ratio, levels of creatinine and alpha-fetoprotein (AFP), and complete blood count (white blood cells, hemoglobin, and platelets). The sensitivity of HCV RNA PCR tests was $15 \mathrm{IU} / \mathrm{mL}$. Additional ALT and AST levels and HCV RNA PCR test results were collected at the time points of treatment weeks 2, 4, end of treatment (EOT), posttreatment weeks 12 and 24, and end of follow-up (EOF). Diagnosis of cirrhosis was based on histology, imaging evidence of cirrhosis and ascites, or endoscopic evidence of esophageal/gastric varices.

\section{Statistical analysis}

Variables were described using number and percentage or mean with the range. The SPSS program was used for statistical analysis. Pearson chi-square, univariate and multivariate analyses were performed to evaluate the association between different variables to ALT and AST normalization during the HCV treatment, with $p$-value $<0.05$ considered statistically significant.

\section{Results}

\section{Baseline characteristics of the study subjects}

In 115 patients, $47.8 \%$ had histological or clinical diagnosis of cirrhosis, with a mean MELD score of 8.8 ; among these patients, $85.5 \%$ were C-P class $A$ and $14.5 \%$ were C-P class $B$, and $14.5 \%$ had hepatic decompensation with ascites and/or hepatic encephalopathy. In 73 (63.5\%) patients who had liver biopsy data, $52.1 \%$ had fibrosis stage $1-2,47.9 \%$, fibrosis stage $3-4$; among these, $69(60 \%)$ patients had steatosis based on imaging and/or biopsy (Table 1 ) and $13(11.3 \%)$ had biopsy proven coexisting NASH. Only 4 (3.5\%) patients had concomitant AIH. HCV distribution is as following: 89 (77.4\%) GT1 (34 GT1a, 31 GT1b, and 24 had no subtype test); 12 (10.4\%) GT2; $9(7.8 \%)$ GT3; and $5(4.4 \%)$ GT4-6.

As shown in Table 1, the mean age was 58.39 (20-85) years. $50.4 \%$ of patients were male. $51.3 \%$ were Caucasian, $20.9 \%$ were Asian, $13.9 \%$ were Hispanic, $5.2 \%$ were AfricanAmerican, and $8.7 \%$ were identified as other race. The mean follow-up was $49(12-122)$ weeks post-treatment. In 45 $(39.1 \%)$ treatment experienced patients, 35 patients (77.8\%) received pegylated interferon (PEG-IFN) with RBV or PEG-IFN monotherapy, 9 patients $(20 \%)$ received PEGIFN/RBV with protease inhibitor, and 1 (2.2\%) received PEG-IFN + RBV with sofosbuvir. For the DAA treatment regimens in this study, there were 66 patients treated with ledipasvir and sofosbuvir +/- RBV, 18 with sofosbuvir + RBV, 6 with $3 D+/-$ RBV, 15 with sofosbuvir and simeprevir, 3 with elbasvir and grazoprevir, 2 with daclatasvir and sofosbuvir, and 5 patients with PEG-IFN + RBV + sofosbuvir.

Mean baseline HCV RNA was log 5.98 (range: 2.65-7.26) $\mathrm{IU} / \mathrm{mL}$. Percent of patients with baseline serum ALT $>40 \mathrm{IU} / \mathrm{L}$ and AST >40 IU/L was $72.2 \%$ and $65.2 \%$, respectively, and $59.1 \%$ had both ALT and AST > 40 IU/L. Mean creatinine was $0.85 \mathrm{mg} / \mathrm{dL}$ (range: $0.3-2.18$ ), mean hemoglobin was 13.78 $\mathrm{g} / \mathrm{dL}$ (range: 8.1-17.2), mean white blood cell count was $5.38 \times 1000 / \mu \mathrm{L}$ (range: $1.7-9.1$ ), and mean platelet count was $169.73 \times 1000 / \mu \mathrm{L}$ (range: $32-467$ ).

In our cohort of patients, percent with baseline serum ALT >40 IU/L and AST >40 IU/L were $72.2 \%$ and $65.2 \%$, respectively, and $59.1 \%$ had both ALT and AST > 40 IU/L. 
Table 1. Baseline characteristics in 115 study subjects

\begin{tabular}{|c|c|}
\hline Characteristic & $n$ (\% or range) \\
\hline Mean age, years & $58.39(20-85)$ \\
\hline Male:female & $58: 57(50.4: 49.6)$ \\
\hline Mean BMI, kg/m² & $27.2(18.1-45.9)$ \\
\hline BMI $>30 \mathrm{~kg} / \mathrm{m}^{2}$ & $30(26.1)$ \\
\hline \multicolumn{2}{|l|}{ Ethnicity } \\
\hline Caucasian & $59(51.3)$ \\
\hline Asian & $24(20.9)$ \\
\hline Hispanic & $16(13.9)$ \\
\hline African American & $6(5.2)$ \\
\hline Others & $10(8.7)$ \\
\hline Treatment-experienced & $45(39.1)$ \\
\hline Clinical diagnosis of cirrhosis & $55(47.8)$ \\
\hline Mean MELD score & $8.8(6-29)$ \\
\hline CPC A & $98(85.5)$ \\
\hline СРC B & $17(14.5)$ \\
\hline Liver biopsy & $73(63.5)$ \\
\hline $\mathrm{F} 1-\mathrm{F} 2$ & $38(52.1)$ \\
\hline F3-F4 & $35(47.9)$ \\
\hline Steatosis & $69(60)$ \\
\hline Coexisting NASH & $13(11.3)$ \\
\hline Coexisting $\mathrm{AIH}$ & $4(3.5)$ \\
\hline \multicolumn{2}{|l|}{ HCV genotype } \\
\hline 1 & $89(77.4)$ \\
\hline 2 & $12(10.4)$ \\
\hline 3 & $9(7.8)$ \\
\hline $4-6$ & $5(4.4)$ \\
\hline ALT $<40 \mathrm{IU} / \mathrm{L}$ & $32(27.8)$ \\
\hline AST $<40 \mathrm{IU} / \mathrm{L}$ & $40(34.8)$ \\
\hline Mean baseline HCV RNA log & $5.98(2.65-7.26)$ \\
\hline Mean baseline AST, IU/L & $67.9(18-244)$ \\
\hline Mean baseline ALT, IU/L & $78.1(12-496)$ \\
\hline Mean baseline AFP, ng/dL & $14.4(1.1-197)$ \\
\hline
\end{tabular}

Abbreviations: AFP, alpha-fetoprotein; AIH, autoimmune hepatitis; ALT, alanine aminotransferase; AST, aspartate aminotransferase; BMI, body mass index; CPC, Child-Pugh class; HCV, hepatitis C virus; MELD, model for end-stage liver disease; $\mathrm{NASH}$, nonalcoholic steatohepatitis.

\section{HCV treatment response and patterns of ALT and AST normalization}

In our cohort of HCV patients who received DAA treatment, serum HCV RNA level declined to $<700 \mathrm{IU} / \mathrm{mL}$ in $100 \%$ and was undetectable in $45.9 \%$ as early as treatment week 2 . For those with remaining detectable HCV RNA, the mean level decreased to 77 (range: 12-630) IU/mL. As shown in Table 2 and Fig. 1, the percentage of undetectable HCV RNA continued increasing to $100 \%$ in EOT.

In this study, normalization was defined by baseline level greater than the upper limit of normal (ULN) (i.e. ALT or AST $\geq 40 \mathrm{IU} / \mathrm{L}$ ) with subsequent decrease to below ULN (i.e. $<40$ IU/L). As shown in Table 2, consistent with virologic response, the ALT and AST normalization started as early as treatment week 2, from $27.8 \%$ and $34.8 \%$ to $85.5 \%$ and $83.9 \%$, respectively. The percentage of the improvement continued to rise in treatment week 4, until posttreatment week 12, up to $97.0 \%$ and $91.8 \%$, respectively. Such ALT and AST normalization remained sustained until EOF. Indeed, there was continuous increase in percentage of ALT and AST normalization from posttreatment week 12 to EOF. Both ALT and AST normalization occurred as early as in treatment week 2, rising from $22.6 \%$ at baseline to $77.4 \%$ and $90.8 \%$ in posttreatment week 12 .

Consistent with ALT or AST normalization, the percentage of both ALT and AST normalization was further increased to 93.0\% at EOF. Fig. 1 shows the dynamic decline of ALT and AST in reference to HCV RNA during and after DAA treatment. However, the overall percentage of both ALT and AST normalization was lower than that of either ALT or AST normalization. Paired sample $t$-test showed that compared to the baseline ALT and AST values, the $p$-values of both ALT and AST declined at each time point, and were all $<0.001$ (i.e. showing statistical significance). In 6 cases with HCV relapse after finishing the DAA treatment, all had EOT ALT and AST normalization, but experienced recurrent ALT and AST elevation after the $\mathrm{HCV}$ relapse.

In 20 cases with pretreatment ALT and AST <40 (range: 12-39) IU/L, 100\% had further decline in both ALT and AST as early as treatment week 2 , which remained in posttreatment week 12 . In 19 of the cases with posttreatment week 12 data, $18 / 19(94.7 \%)$ had both ALT and AST decline to <25 IU/L. One case had ALT of $37 \mathrm{IU} / \mathrm{L}$ and AST of $29 \mathrm{IU} / \mathrm{L}$, but this carried a diagnosis of coexisting NASH.

The differences in ALT normalization between male and female patients were analyzed. In 19 patients with baseline ALT $<40$ IU and posttreatment week 12 follow-up, 5/8 $(62.5 \%)$ male patients had baseline ALT <30 (range: $12-29$ ) IU/L and $1 / 11$ ( $9 \%$ ) female patients had ALT <19 (range: 13-16) IU/L. At posttreatment week 12, 8/8 (100\%) males had ALT <30 (range: $10-29$ ) IU/L and 9/11 (81.8\%) females had ALT <19 (range: 9-17) IU/L.

In 17 patients with liver biopsy diagnosis of NASH or AIH (NASH, $n=13 ; \mathrm{AIH}, n=4$ ), 93.3\%, $86.7 \%$ and $86.7 \%$ had ALT, AST and both ALT and AST normalization in posttreatment week 12. Fig. 1 shows the dynamic changes of mean ALT and AST in association with serum HCV RNA changes, from baseline to EOF. Both ALT and AST normalization was well associated with the clearance of HCV replication.

\section{Variables associated with ALT and AST normalization}

As shown in Table 3, univariate analysis was performed to evaluate factors associated with both ALT and AST normalization to $<40 \mathrm{IU} / \mathrm{L}$ at posttreatment weeks 12 . The variables of age $>50$ years, ethnicity, presence of $\mathrm{NASH}$ or $\mathrm{AIH}$, body mass index $>30 \mathrm{~kg} / \mathrm{m}^{2}$, and baseline AFP elevation to $>5.5$ $\mathrm{ng} / \mathrm{dL}$ were not associated with ALT and AST normalization to $<40 \mathrm{IU} / \mathrm{L}$. However, HCV GT 1, HCV RNA of < 500 IU in treatment week 2, C-P score of $<6$, and MELD score of $<10$ were significantly associated with both ALT and AST normalization to $<40 \mathrm{IU} / \mathrm{L}$ in posttreatment week 12 . Multivariate analysis confirmed that baseline MELD score of $<10$ was significantly associated with both ALT and AST normalization at posttreatment week 12, independent to HCV RNA $<500 \mathrm{IU} / \mathrm{mL}$ at 
Huynh T. et al: HCV clearance and rapid ALT/AST normalization

Table 2. Dynamic changes in serum HCV RNA, ALT, AST, and both ALT and AST to $<40$ IU/L (\%) from baseline to end of follow-up

\begin{tabular}{|c|c|c|c|c|c|c|c|c|}
\hline & Baseline & Wk2 & Wk4 & Wk8 & EOT & PRx Wk12 & PRx Wk24 & EOF \\
\hline $\begin{array}{l}\text { HCV RNA } \\
<15 \mathrm{IU} / \mathrm{mL}\end{array}$ & 0.00 & $\begin{array}{l}45.9 \\
(28 / 61)\end{array}$ & $\begin{array}{l}78.2 \\
(79 / 101)\end{array}$ & $\begin{array}{l}95.3 \\
(82 / 86)\end{array}$ & $\begin{array}{l}100 \\
(100 / 100)\end{array}$ & $100(95 / 95)$ & $100(67 / 67)$ & $\begin{array}{l}100 \\
(92 / 92)\end{array}$ \\
\hline ALT $<40 \mathrm{IU} / \mathrm{L}$ & $\begin{array}{l}27.8 \\
(32 / 115)\end{array}$ & $\begin{array}{l}85.5 \\
(53 / 62)\end{array}$ & $\begin{array}{l}92.9 \\
(91 / 98)\end{array}$ & & $\begin{array}{l}91.3 \\
(95 / 104)\end{array}$ & $97.0(95 / 98)$ & $96.1(74 / 77)$ & $\begin{array}{l}97.4 \\
(112 / 115)\end{array}$ \\
\hline AST $<40 \mathrm{IU} / \mathrm{L}$ & $\begin{array}{l}34.8 \\
(40 / 115)\end{array}$ & $\begin{array}{l}83.9 \\
(52 / 62)\end{array}$ & $\begin{array}{l}91.8 \\
(90 / 98)\end{array}$ & & $\begin{array}{l}91.3 \\
(95 / 104)\end{array}$ & $91.8(90 / 98)$ & $94.8(73 / 77)$ & $\begin{array}{l}94.8 \\
(109 / 115)\end{array}$ \\
\hline $\begin{array}{l}\text { Both ALT and } \\
\text { AST }<40 \mathrm{IU} / \mathrm{L}\end{array}$ & $\begin{array}{l}22.6 \\
(26 / 115)\end{array}$ & $\begin{array}{l}77.4 \\
(48 / 62)\end{array}$ & $\begin{array}{l}85.7 \\
(84 / 98)\end{array}$ & & $\begin{array}{l}85.6 \\
(89 / 104)\end{array}$ & $90.8(89 / 98)$ & $94.8(73 / 77)$ & $\begin{array}{l}93.0 \\
(107 / 115)\end{array}$ \\
\hline
\end{tabular}

Abbreviations: ALT, alanine aminotransferase; AST, aspartate aminotransferase; EOF, end of follow-up; EOT, end of treatment; PRx Wk, posttreatment week; Wk, treatment week.

treatment week 2 and C-P score of $<6$, although C-P score of $<6$ was also almost significantly associated.

\section{Discussion}

ALT and AST elevation is a biochemical surrogate for hepatocytic injury and a typical presentation of CHC. Studies have demonstrated persistent elevation of ALT and AST is associated with $\mathrm{CHC}$ progression and increasing risk for cirrhosis, and indication for HCV treatment. ${ }^{7-10}$ For instance, ALT elevation was reported to be present in $75 \%$ of patients with CHC. ${ }^{11}$ In our cohort of patients, baseline serum ALT $>40$ $\mathrm{IU} / \mathrm{L}$ and AST $>40 \mathrm{IU} / \mathrm{L}$ were found in $72.2 \%$ and $65.2 \%$ of the patients, respectively, and $59.1 \%$ had both ALT and AST $>40 \mathrm{IU} / \mathrm{L}$, consistent with previous reports.

DAA has become the standard of HCV treatment, with very high SVR rates demonstrated by clinical trials and real-world data. ${ }^{12-14}$ However, biochemical response and factors related

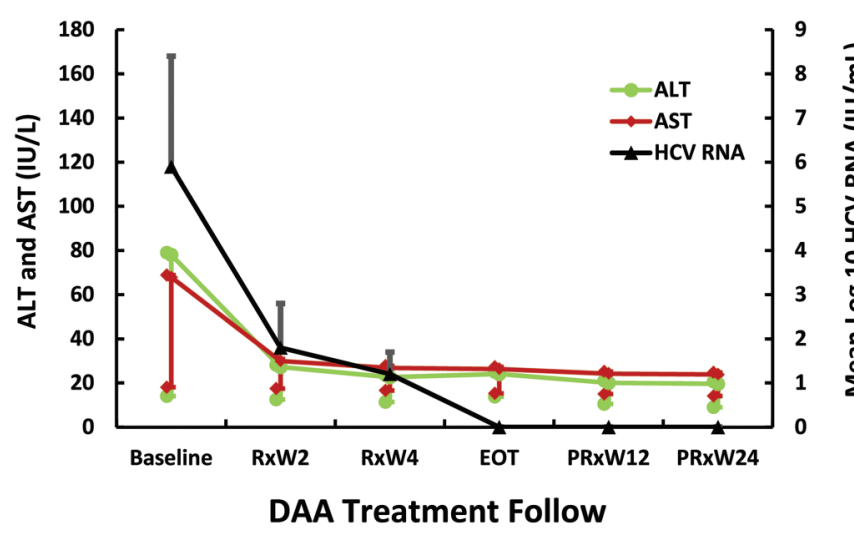

Fig. 1. Dynamic changes of mean HCV RNA, ALT and AST during and after DAA treatment. The dynamic changes of ALT (IU/L), AST (IU/L) and HCV RNA $(\mathrm{IU} / \mathrm{mL}$ ) including standard deviations are shown on the $y$-axis. Baseline and time line of the DAA treatment and of the posttreatment follow-up (by week, or W) are shown on the $x$-axis. Both mean ALT and AST decline and normalization rapidly following HCV RNA decline and undetectability occurred as early as DAA treatment week 2, indicating HCV clearance resulted in ALT and AST normalization in HCVinfected patients. Paired sample $t$-test showed that compared to the baseline ALT and AST values, the $p$-values of both ALT and AST decline at each time point were all $<0.001$.

Abbreviations: ALT, alanine aminotransferase; AST, aspartate aminotransferase; DAA, direct-acting antiviral; EOT, end of treatment; HCV, hepatitis $C$ virus; PRxW, posttreatment week; RxW, treatment week. to biochemical response during and after DAA therapy have not been previously reported. The present study assessed ALT and AST dynamic changes during and after DAA treatment in association with $\mathrm{HCV}$ clearance in patients with $\mathrm{CHC}$. For a long time, HCV has been considered nonpathogenic, and that it is the host immune response to HCV proteins that caused hepatocytic injury. ${ }^{15-17}$ However, we found that in most of the patients, HCV decline and clearance occurred as early as 2 weeks after initiating the DAA treatment. Consistent with this, normalization of ALT and AST elevation occurred as early as 2 weeks after DAA treatment. More specifically, at week 2 of DAA treatment, serum HCV RNA declined to $<700 \mathrm{IU} / \mathrm{mL}$ in $100 \%$ and was undetectable in $45.9 \%$ of the patients, and $85.5 \%$ and $83.9 \%$ experienced ALT and AST normalizations, respectively, and $77.4 \%$ experienced both ALT and AST normalization. Therefore, for the first time, we demonstrated the benefit of DAA treatment not only for HCV RNA clearance but also for normalization of ALT and

Table 3. Univariate and multivariate analysis for factors associated with both ALT and AST <40 IU/L at post-DAA Rx week 12

\begin{tabular}{lll}
\hline Variable & $\begin{array}{l}\text { Univariate } \\
\text { analysis } \\
p \text {-value }\end{array}$ & $\begin{array}{l}\text { Multivariate } \\
\text { analysis } \\
p \text {-value (LR) }\end{array}$ \\
\hline $\begin{array}{l}\text { Age }>50 \text { years } \\
\text { Asian ethnicity }\end{array}$ & 0.242 & \\
$\begin{array}{l}\text { Presence of } \\
\text { NASH or AIH }\end{array}$ & 0.239 & \\
BMI $>30 \mathrm{~kg} / \mathrm{m}^{2}$ & 0.591 & \\
$\begin{array}{l}\text { Baseline AFP } \\
>5.5 \text { ng/dL }\end{array}$ & 0.296 & \\
$\begin{array}{l}\text { Presence of } \\
\text { cirrhosis }\end{array}$ & 0.523 & \\
GT 1 vs. non-1 & 0.362 & $0.250(2.77)$ \\
HCV RNA <500 IU & 0.010 & $0.148(3.82)$ \\
at Rx Wk 2 & 0.028 & $0.063(8.32)$ \\
CPC score $<6$ & 0.022 & $0.044(9.00)$ \\
MELD score $<10$ & 0.035 & \\
\hline
\end{tabular}

Abbreviations: AFP, alpha-fetoprotein; AIH, autoimmune hepatitis; BMI, body mass index; CPC, Child-Pugh class; DAA, direct-acting antiviral; GT, genotype; $\mathrm{HCV}$, hepatitis C virus; LR, likelihood ratio; MELD, model for end-stage liver disease; $\mathrm{NASH}$, nonalcoholic steatohepatitis; Rx, treatment; Wk, week. 
AST - traditional biochemical surrogates of liver injury - as early as 2 weeks after initiating the DAA treatment.

Both ALT and AST improvement continued with the course of DAA treatment and HCV RNA clearance. On the other hand, in 6 cases with baseline elevation of both ALT and AST and treatment-induced ALT and AST normalizations but posttreatment virologic relapse, all developed recurrent elevation of both ALT and AST once the virological relapse had occurred. As both ALT and AST elevation is the surrogate for hepatocytic injury, such close correlation of ALT and AST improvement with HCV RNA clearance also provides strong clinical evidence that HCV may be directly pathogenic, causing hepatocytic injury. If this finding is further confirmed by large prospective studies and the underlying mechanism is determined, our findings will lend support to the theory that HCV could be directly pathogenic in HCV-related pathogenesis, in contrast to the traditional concept.

We also found that all patients with baseline ALT and AST $<40$ IU had further ALT and AST decline right after HCV clearance that remained to posttreatment week 12 . Modified ALT ULN (i.e. $<30 \mathrm{IU} / \mathrm{L}$ for males and <19 IU/L for females) has been included for hepatitis $B$ treatment guidance, ${ }^{18}$ but no studies for such with HCV infection have been reported. Another interesting finding of the present study is that in patients with pretreatment ALT of $<40 \mathrm{IU} / \mathrm{L}$, DAA-mediated $\mathrm{HCV}$ clearance resulted in further ALT decline. Indeed, $100 \%$ of male patients had ALT improved to $<30 \mathrm{IU} / \mathrm{L}$, and $81.8 \%$ of female patients had it to $<19 \mathrm{IU} / \mathrm{L}$. Our findings indicated that the current ALT ULN of $40 \mathrm{IU} / \mathrm{L}$ may underestimate HCV-mediated liver injury. Further larger sample studies will be needed to reconfirm our above findings.

It is well known that other kinds of liver diseases or liver injury may coexist with $\mathrm{CHC}$, contributing to progression of liver injury and increasing risk for cirrhosis. ${ }^{19,20}$ In our study, $60 \%$ of patients had steatosis by imaging ultrasound or computerized tomography, 13 cases (11.3\%) had liver biopsy-confirmed diagnosis of NASH, and 4 cases (3.5\%) had pathologic diagnosis of coexisting $\mathrm{AIH}$. We found that in the $17 \mathrm{CHC}$ patients with histologically-confirmed coexistence of NASH or AIH, $93 \%$ and $80 \%$ had baseline ALT or AST elevation, respectively. The rates of ALT and AST normalization were comparable to those in patients without NASH or AIH. Univariate analysis also indicated the presence or absence of NASH, and AIH was not associated with ALT and AST normalization. Although the sample size was small, our results indicated that in those with $\mathrm{CHC}$ and coexisting $\mathrm{NASH}$ or $\mathrm{AIH}, \mathrm{HCV}$-mediated hepatocytic injury is the main driving force for liver injury. Further larger studies are needed to confirm these findings.

The dynamics of normalization were similar for ALT and AST, but the normalization rate was slightly lower for AST than ALT. Normalization of both ALT and AST was not associated with the degree of baseline elevation. For instance, in patients with baseline ALT of $>100 \mathrm{IU} / \mathrm{mL}, 93 \%$ had ALT normalization at EOF. In general, the rate of normalization of both ALT and AST was slightly lower than that of either ALT or AST normalization. We also found that, like serum HCV RNA, the normalization of ALT and AST at posttreatment week 12 was sustained, indicating the possibility to use ALT and AST tests as replacements for the expensive HCV RNA PCR test, and to follow DAA treatment response. If further confirmed, this will be, perhaps, particularly valuable in areas where frequent HCV RNA PCR testing is not affordable. Another interesting finding of our study was that the rate of normalization of both
ALT and AST was further improved from posttreatment week 12 to $\mathrm{EOF}$, from $90.8 \%$ to $93.0 \%$.

We determined the variables associated with normalization of both ALT and AST. Univariate analysis indicated that HCV GT 1 versus non-1, HCV RNA of $<500 \mathrm{IU} / \mathrm{mL}$ in treatment week 2, C-P score of $<6$, and MELD score of $<10$, but not age, ethnicity, presence of NASH or AIH, cirrhosis, obesity, elevated serum AFP, or cirrhosis were significantly associated with normalization of both ALT and AST to $<40 \mathrm{IU} / \mathrm{L}$ in posttreatment week 12 . Multivariate analysis confirmed that baseline MELD score of $<10$ was significantly associated with both ALT and AST normalization at posttreatment week 12, independent to HCV RNA $<500 \mathrm{IU} / \mathrm{mL}$, HCV GT 1, and C-P score. This indicated that absence of advanced cirrhosis (MELD <10) predicts normalization of both ALT and AST, i.e. sustained biochemical response, and that is well correlated to SVR12.

In conclusion, by assessing the dynamic patterns of normalization of both ALT and AST during and after DAA treatment for HCV infection, our study provided evidence that (1) normalization of both ALT and AST is well associated with sustained HCV clearance, which could be a potential biochemical marker to follow HCV treatment responses; (2) rapid normalization of both ALT and AST followed HCV clearance, indicating that HCV might be directly pathogenic, causing hepatocytic injury; and, (3) the current ALT ULN of 40 IU might under-estimate HCV-mediated liver injury.

\section{Conflict of interest}

Dr. $\mathrm{Hu}$ is on the Speaker Bureau for Gilead and Merck. The others have no conflict of interests related to this publication.

\section{Author contributions}

Study design $(\mathrm{KQH})$, data collection and analysis $(\mathrm{KQH}, \mathrm{TH}$, $\mathrm{JZ})$, manuscript preparation $(\mathrm{TH}, \mathrm{JZ})$, final writing and editing of the manuscript $(\mathrm{KQH})$.

\section{References}

[1] Gower E, Estes C, Blach S, Razavi-Shearer K, Razavi H. Global epidemiology and genotype distribution of the hepatitis C virus infection. J Hepatol 2014; 61:S45-S57. doi: 10.1016/j.jhep.2014.07.027.

[2] Edlin BR, Eckhardt BJ, Shu MA, Holmberg SD, Swan T. Toward a more accurate estimate of the prevalence of hepatitis $C$ in the United States. Hepatology 2015;62:1353-1363. doi: 10.1002/hep.27978.

[3] Chen SL, Morgan TR. The natural history of hepatitis C virus (HCV) infection. Int J Med Sci 2006;3:47-52. doi: 10.7150/ijms.3.47.

[4] Zhang J, Nguyen D, Hu KQ. Chronic hepatitis C virus infection: A review of current direct-acting antiviral treatment strategies. N Am J Med Sci (Boston) 2016;9:47-54.

[5] Simmons B, Saleem J, Heath K, Cooke GS, Hill A. Long-term treatment outcomes of patients infected with hepatitis $C$ virus: A systematic review and meta-analysis of the survival benefit of achieving a sustained virological response. Clin Infect Dis 2015;61:730-740. doi: 10.1093/cid/civ396.

[6] Nahon P, Bourcier V, Layese R, Audureau E, Cagnot C, Marcellin P, et al. Eradication of hepatitis $C$ virus infection in patients with cirrhosis reduces risk of liver and non-liver complications. Gastroenterology 2017;152:142-156.e2. doi: 10.1053/j.gastro.2016.09.009.

[7] Attar BM, Van Thiel DH. Hepatitis C virus: A time for decisions. Who should be treated and when? World J Gastrointest Pharmacol Ther 2016;7:33-40. doi: 10.4292/wjgpt.v7.i1.33.

[8] Lynch SM, Wu GY. Hepatitis C virus: A review of treatment guidelines, costeffectiveness, and access to therapy. J Clin Transl Hepatol 2016;4:310-319. doi: $10.14218 /$ JCTH.2016.00027.

[9] González-Grande R, Jiménez-Pérez M, González Arjona C, Mostazo Torres J. New approaches in the treatment of hepatitis C. World J Gastroenterol 2016; 22:1421-1432. doi: 10.3748/wjg.v22.i4.1421. 
[10] Zampino R, Marrone A, Restivo L, Guerrera B, Sellitto A, Rinaldi L, et al. Chronic HCV infection and inflammation: Clinical impact on hepatic and extra-hepatic manifestations. World J Hepatol 2013;5:528-540. doi: 10.4254/ wjh.v5.i10.528.

[11] Marcellin P, Levy S, Erlinger S. Therapy of hepatitis C: Patients with normal aminotransferase levels. Hepatology 1997;26;133S-136S. doi: 10.1002/hep. 510260723.

[12] Younossi ZM, Park H, Gordon SC, Ferguson JR, Ahmed A, Dieterich D, et al. Real-world outcomes of ledipasvir/sofosbuvir in treatment-naive patients with hepatitis C. Am J Manag Care 2016;22:SP205-SP211.

[13] Ioannou GN, Beste LA, Chang MF, Green PK, Lowy E, Tsui JI, et al. Effectiveness of sofosbuvir, ledipasvir/sofosbuvir, or paritaprevir/ritonavir/ombitasvir and dasabuvir regimens for treatment of patients with hepatitis $C$ in the veterans affairs national health care system. Gastroenterology $2016 ; 151$ : 457-471.e5. doi: 10.1053/j.gastro.2016.05.049.

[14] Terrault NA, Zeuzem S, Di Bisceglie AM, Lim JK, Pockros PJ, Frazier LM, et al. Effectiveness of ledipasvir-sofosbuvir combination in patients with hepatitis $C$ virus infection and factors associated with sustained virologic response. Gastroenterology 2016;151:1131-1140.e5. doi: 10.1053/ j.gastro.2016.08.004
[15] Zhang X, Dou J, Germann MW. Characterization of the cellular immune response in hepatitis C virus infection. Med Res Rev 2009;29:843-866. doi: 10.1002/med.20157.

[16] Sun J, Rajsbaum R, Yi M. Immune and non-immune responses to hepatitis C virus infection. World J Gastroenterol 2015;21:10739-10748. doi: 10.3748/ wjg.v21.i38.10739.

[17] Neuman MG, Sha K, Esguerra R, Zakhari S, Winkler RE, Hilzenrat N, et al. Inflammation and repair in viral hepatitis C. Dig Dis Sci 2008;53:1468-1487. doi: 10.1007/s10620-007-0047-3.

[18] Terrault NA, Bzowej NH, Chang KM, Hwang JP, Jonas MM, Murad MH. AASLD guidelines for treatment of chronic hepatitis B. Hepatology 2016;63:261-283. doi: 10.1002/hep.28156.

[19] Patton HM, Patel K, Behling C, Bylund D, Blatt LM, Vallée M, et al. The impact of steatosis on disease progression and early and sustained treatment response in chronic hepatitis C patients. J Hepatol 2004;40:484-490. doi: 10.1016/j.jhep.2003.11.004.

[20] Dyal HK, Aguilar M, Bhuket T, Liu B, Holt EW, Torres S, et al. Concurrent obesity, diabetes, and steatosis increase risk of advanced fibrosis among HCV patients: A systematic review. Dig Dis Sci 2015;60:2813-2824. doi: $10.1007 / \mathrm{s} 10620-015-3760-3$. 